\title{
Pathogenic Factors and Immunological Treatment of Cervical Cancer
}

\author{
QIN QINGYING ${ }^{1, a}$, HE WENYUAN ${ }^{1}$, WANG GUXIAN ${ }^{1}$ \\ ${ }^{1}$ QUJING MEDICAL COLLEGE
}

\begin{abstract}
This paper attempts to analyze the related factors of cervical cancer combined with immunotherapy, taking cervical cancer patients as the experimental object, through the establishment of cervical cancer model, combined with natural immune agonists, immune activated monoclonal antibody and HPV polypeptide to explore its prevention or treatment of cervical cancer immune research. The results showed that the number of people with bad habits was more, and the incidence rate of cervical cancer was relatively high. However, for those without formal work, the number of cases of bad sexual behavior is more, and HPV transmission is easier, which also provides convenience for the occurrence of cervical cancer. The mechanism of action of tumor therapeutic vaccine is different from that of cytotoxic drugs. Its particularity lies in that the induction of specific anti-tumor immune response needs a certain period of time before it can be transformed into clinical effect; and patients with recurrence or metastasis usually receive multiple rounds of treatment, which may affect the immune system and reduce the efficacy of the vaccine.
\end{abstract}

\section{Introduction}

Cervical cancer is the most common malignancy in female genital tract. Due to the popularity of cervical cytology screening, the improvement of social and economic level and the change of lifestyle, the incidence rate and mortality rate of cervical cancer in most developed countries showed a downward trend. However, for patients with advanced stage, uncontrollable after treatment or recurrence or metastasis, the effect of existing treatment is not satisfactory.

Cervical cancer is a serious threat to women's health and one of the most common malignant tumors. Gagliard analyzed 118 cases of cervical cancer from Uganda, and determined the specific differences of HPV clade in tumor DNA methylation, regulatory region related histone markers, gene expression and pathway imbalance [2-3]. Yang found that two cervical cancer cell lines HeLa and $\mathrm{SiHa}$ did not respond to the cytotoxicity of TNF - $\alpha$. However, pten-130b down regulates the expression of tumor specific mir-130b. The cytotoxicity to TNF - $\alpha$ of sensitized cells by blocking mir-130b function or overexpression of PTEN gene. Yang's experiment showed that mir-130b inhibited PTEN gene expression by directly binding with 3'UTR of PTEN mRNA. These data suggest that mir-130b expression is a target of inhibition, which makes tumor cells more sensitive to the toxic effects of TNF $-\alpha[4-5]$.

In this paper, we followed the domestic and foreign research on cervical cancer immunology, and further explored the latest achievements of immunotherapy and prevention on the basis of understanding the pathogenic factors and pathogenesis of cervical cancer. In this study, we established a model of cervical cancer, combined with natural immune agonists, immune activated monoclonal antibody and HPV16E7 polypeptide to explore the immunological research of its prevention or treatment of cervical cancer. By observing the growth of tumor after immunotherapy to investigate its preventive effect. This paper analyzes the correlation between tigit and $\mathrm{T}$ cell specific immune response in cervical cancer, which can provide clues for immunotherapy of cervical cancer.

\section{The Pathogenesis of Cervical Cancer and Immunological Treatment}

\subsection{Risk Factors of Cervical Cancer}

The incidence of cervical cancer is the result of multi factor synergy. Generally, it can be divided into carcinogenic factors and carcinogenic factors. The former acts on cervical epithelial reserve cells to produce gene mutations, such as HPV binding into the cell chromosome, and then further leads to carcinogenesis under the action of cancer promoting factors. The factors promote and influence each other: premature delivery and Prolificacy are easy to cause cervical endometrium eversion and erosion; early marriage and frequent sexual life and disorder can easily cause cervical erosion, and strengthen the role of virus and foreskin scale in promoting cancer. Especially erosion is not only the result of the above factors, but also the internal basis of the growth, reproduction and cell transformation of HPV or HSV [6-7].

HPV is a small double stranded closed loop DNA virus. 
Its gene fragment is divided into three regions[8]: upstream non coding region, early coding region and late coding region. The early coding region includes E1 $\sim$ E7, the coding products mainly regulate viral DNA replication, viral RNA transcription, cytoskeleton recombination and cell transformation; E6 and E7 are high-risk factors of CIN and cervical cancer, E6 can induce p53 degradation, E7 can inhibit the anti-cancer function of $\mathrm{Rb}$, so it is an important target gene in the research of HPV carcinogenesis mechanism and the development of vaccine against HPV related cancer. The late coding region includes L1 and L2, and the encoded products are structural elements of viral capsid, in which L1 is the major capsid protein and L2 is the secondary capsid protein. If the body can produce neutralizing antibodies against HPV capsid proteins L1 and L2 after HPV infection, HPV infection can be prevented[9-10].

The incidence of cervical cancer is related to social factors, which have a certain synergistic effect on the occurrence of cervical cancer. Among these complex social factors, low education level is the most closely related risk factor of cervical cancer. Due to the limitation of education level, these women's economic income is relatively low, living conditions are relatively poor, personal health care awareness is also not up to date, many women have poor awareness of physical examination, rarely do gynecological examination, coupled with these women are more likely to marry prematurely, leading to increased risk of cervical cancer. In general, the prevalence of cervical cancer in rural areas is higher than that in urban areas, and that in economically backward areas is higher than that in economically developed areas. Patients with different education levels have different cognitive ability of disease, which leads to different impact on the quality of life. The higher the education level, the higher the quality of life of patients.

\subsection{Immunotherapy}

The traditional treatment of cervical cancer only has a certain effect on early patients, and the treatment of trauma, can not prevent HPV reinfection. For patients who have not been infected with HPV, vaccination is the best choice. Early intervention and early treatment are particularly important for patients who have been infected with HPV. HPV infection is often accompanied by serious side effects in the treatment process. The immune system of patients with cervical cancer who have undergone surgery, radiotherapy and chemotherapy is in a state of depression. After vaccination, the body can not produce ideal antigenspecific immune response. There are many kinds of therapeutic vaccines, including polypeptides, proteins, virus vectors and plasmid vectors. Unlike preventive vaccines, therapeutic vaccines aim to establish a cellmediated immune response to remove infected cells. Polypeptide therapeutic HPV vaccine breaks the immune tolerance of patients through specific immune stimulation, and produces specific cellular and humoral immune responses against $\mathrm{HPV}$, so as to eliminate or inhibit the virus and treat diseases. E6 / E7 is an important oncogenic protein of HPV. E6 / E7 based on cellular immune response may cause HPV associated precancerous lesions or tumor cells to express these viral proteins. Therefore, the sustained expression of E6 / E7 in the infected site makes them an ideal target for therapeutic vaccines. Although therapeutic peptide vaccines have the disadvantages of low immunogenicity, specific MHC epitopes and adjuvants, they have the advantages of high stability, strong safety and easy production.

DNA vaccine is a kind of vaccine designed according to gene. As an immunogen, it is safe in theory. The principle of its production is to recombine a gene with antigenicity into eukaryotic expression vector according to a certain operation procedure and in a specific experimental environment. Then it is injected into the human body, and the host cells are transfected into the human body to induce an adaptive immune function. Through the comparative study of gene immunization of the same antigen from different inoculation ways, it was found that when the body injected DNA plasmid to the target tissue point, a short electric pulse could promote the permeability of cell membrane, promote the absorption of plasmid DNA plasmid to a certain extent, and further promote the expression of plasmid DNA. Due to the higher efficiency of tissue transfection, the immune effect of CD8 + T lymphocyte activation is higher than that of intramuscular injection and intradermal gene gun. However, when the vaccine contains high integrity E6 / E7 gene fragment, it may lead to the risk of cell mutation transformation, which will lead to HLA expression by mutating the p53 / RB site of E6 / E7, but will not further produce favorable antigen protein to avoid this harm. For cervical cancer patients who have completed the standard chemoradiotherapy and can not be operated on, if the electroporation vaccine is injected, the tolerance, safety and immunogenicity of the vaccine are better, which has better clinical application potential.

\section{Experiments Materials and Methods}

\subsection{Experimental Materials}

In this paper, 50 cases of cervical cancer patients in a hospital of our city from September 2017 to September 2019 were selected as the observation group, aged between 25 and 50 years old; 50 cases of healthy people in the same period were selected as the control group, aged from 24 to 48 years old; both groups were married; there was no significant difference in gender and age between the two groups.

\subsection{Experimental Methods}

Two groups of patients were investigated by self-designed questionnaire, accompanied by specialized nursing staff to fill in the relevant information, the questionnaire mainly includes education level, monthly income, lifestyle, sexual habits, medical history, etc. The patients in the observation group were collected specimens to observe the clinicopathological characteristics of cervical cancer. Pelvic examination, cervical cytology examination and biopsy were feasible to ensure that there were no other 
malignant tumors in the patients. If necessary, uterine curettage could be used.

Firstly, the PBMC and til isolated from tissue samples were used as blank control. Bv510 dye was added to the remaining cells and incubated in the refrigerator. Then, the cells were washed twice with precooled FACS. After centrifugation, the cells were resuspended with FACS, and antibody was added. After vortex mixing, the cells were incubated in the refrigerator. Finally, the cells were washed twice with the precooled FACS. After centrifugation, FACS was added to fix the cells, Wait for the machine to be tested by flow instrument. Flowjo software was used to analyze the experimental results,

\section{Analysis of the Pathogenic Factors and Immunological Treatment of Cervical Cancer}

\subsection{Analysis of Related Influencing Factors of Cervical Cancer}

The difference was statistically significant between the observation group and the control group. The related influencing factors of cervical cancer are shown in Table 1.

Table1. Related influencing factors of cervical cancer (\%)

\begin{tabular}{cccc}
\hline & & Observation group & Control group \\
\hline Smoking history & Have & 61.43 & 22.54 \\
Childbirth before the & No & 39.21 & 78.29 \\
age of 20 & Yes & 27.57 & 11.42 \\
& No & 72.35 & 89.35 \\
Parity & $\geq 3$ & 41.24 & 14.48 \\
& $<3$ & 59.08 & 85.73 \\
History of abortion & $\geq 2$ & 39.32 & 11.35 \\
History of family & $<2$ & 61.43 & 89.22 \\
related illnesses & Have & 50.67 & 22.04 \\
Sexual partner & No & 49.25 & 78.37 \\
Age at first sex & $\geq 3$ & 21.26 & 7.36 \\
& $<3$ & 78.84 & 93.31 \\
& $\leq 20$ & 39.52 & 18.25 \\
\hline
\end{tabular}

The rate of smoking history, fertility rate before the age of 20 , parity number greater than or equal to three, induced abortion more than or equal to twice, family history of related diseases, number of sexual partners greater than or equal to 3 , age of first sexual life less than or equal to 20 years old in the observation group were higher than those in the control group, the difference was statistically significant. The analysis of related influencing factors of cervical cancer is shown in Figure 1.

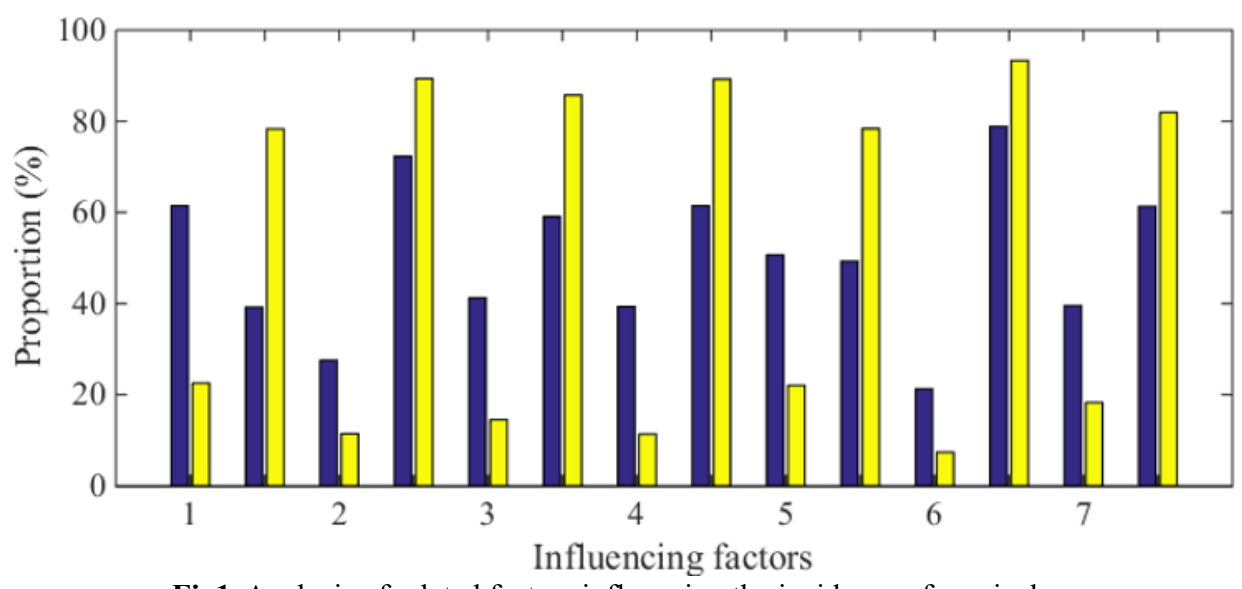

Fig1. Analysis of related factors influencing the incidence of cervical cancer

Comparing the general data of two groups of patients, it was found that cervical cancer incidence was concentrated in low education, low income and no formal work. Combined with another result in this article, the incidence of seven factors in the observation group was higher than that in the control group. It can be found that there are more people with low living habits and low educational background, and the incidence rate of cervical cancer is relatively high. However, for those without formal work, the number of cases of bad sexual behavior is more, and HPV transmission is easier, which also provides convenience for the occurrence of cervical cancer. 


\subsection{Immunotherapy Analysis}

Logistic test showed that the age of first sexual intercourse less than 20 years old, chronic cervicitis and HPV infection were the high risk factors of cervical cancer. Logistic regression analysis of risk factors for cervical cancer is shown in Figure 2.

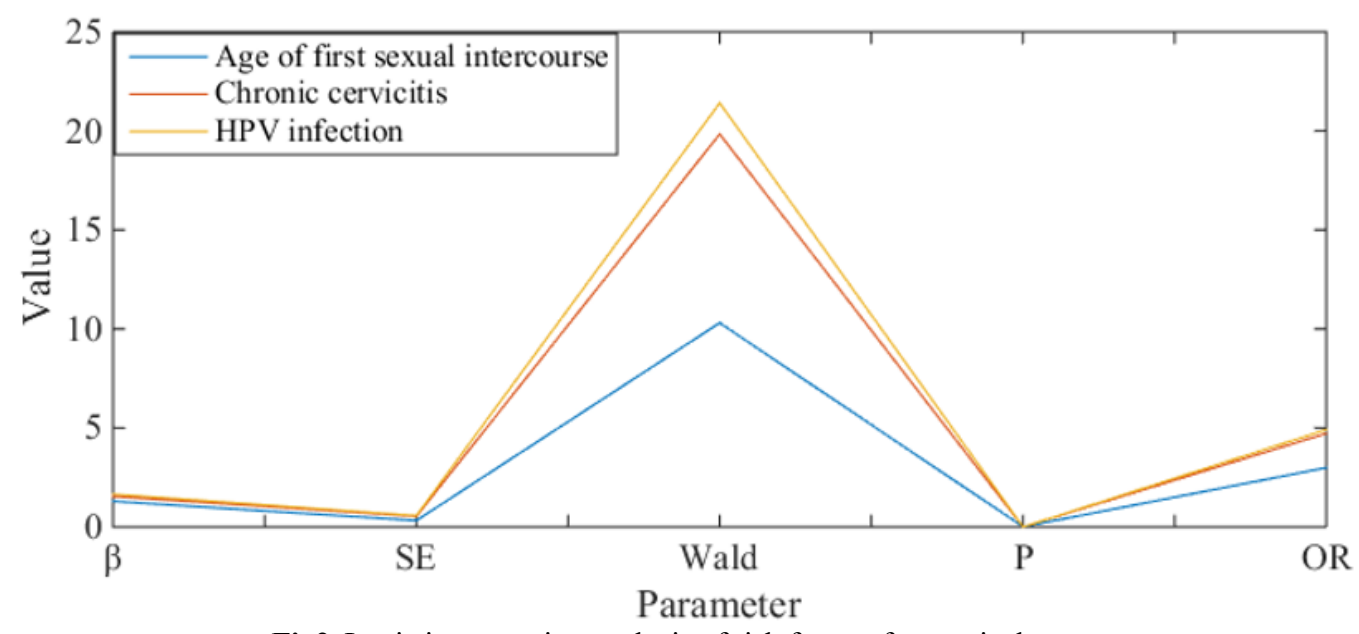

Fig2. Logistic regression analysis of risk factors for cervical cancer

The expression level of tigit on CD4 $+\mathrm{T}$ cells in cervical cancer tissues was different in age group and menopause group. The difference of tigit expression frequency between the observation group and the control group was statistically significant, and the expression level of the former was lower than that of the latter. The expression level of tigit on CD4 $+\mathrm{T}$ cells in postmenopausal group was lower than that in non menopausal group, the difference was statistically significant. The expression of tigit on $\mathrm{CD} 8+\mathrm{T}$ was significantly different in the gross types of tumors. No significant difference was found in the expression of tigit on $\mathrm{T}$ cells among patients grouped according to family history of cancer, tumor size, differentiation degree, clinical stage, squamous cell carcinoma antigen or pelvic lymph node metastasis. The mechanism of action of tumor therapeutic vaccine is different from that of cytotoxic drugs. Its particularity is that it takes a certain time to induce specific anti-tumor immune response, And patients with recurrence or metastasis usually receive multiple rounds of treatment, which may affect the immune system and reduce the efficacy of the vaccine. Therefore, it is necessary to weigh the advantages and disadvantages of patients with advanced tumor, or patients with less tumor load or no obvious residual tumor in remission stage.

\section{Conclusions}

Prevention and control of cervical cancer is a social problem, and its influencing factors are very extensive. Therefore, prevention should be taken from the comprehensive aspect in daily life. Maintaining a good lifestyle is an important measure to reduce the incidence rate of cervical cancer. The etiology of cervical cancer is complex, but the relationship between HPV and cervical cancer is clear. Immunotherapy represented by vaccine is undergoing preclinical or clinical trials, but it is developing in a good direction. In the near future, the existing problems of therapeutic vaccines will be overcome, which will greatly improve the prognosis of cervical cancer.There are still some deficiencies in this paper, such as the small sample size, no analysis of the dynamic changes of tigit during the treatment process, and no further analysis of the relationship between the expression of tigit and the prognosis of cervical cancer. Therefore, it is necessary to expand the sample size for further research and analysis, so as to provide theoretical basis for immunotherapy of cervical cancer.

\section{References}

1. Flanagan M B . Primary High-Risk Human Papillomavirus Testing for Cervical Cancer Screening in the United States: Is It Time?[J]. Archives of pathology \& laboratory medicine, 2018, 142(6):688692.

2. Gagliardi A, Porter V L, Zong Z, et al. Analysis of Ugandan cervical carcinomas identifies human papillomavirus clade-specific epigenome and transcriptome landscapes[J]. Nature Genetics, 2020, 52(8):800-810.

3. Hee L Y, Kyung K M , Young M H , et al. Proteomic Analysis of Pelvic Autonomic Nerve in Nervesparing Radical Hysterectomy for Cervical Carcinoma.[J]. Cancer genomics \& proteomics, 2018, 15(4):337-342.

4. Yang L, Wang Y, Shi S, et al. TheTNF - $\alpha$ - induced expression of $\mathrm{miR}-130 \mathrm{~b}$ protects cervical cancer cells from the cytotoxicity ofTNF $-\alpha[\mathrm{J}]$. FEBS Open Bio, 2018, 8(4):614-627.

5. Guo M , Huang E, Liu X, et al. Volumetric Modulated Arc Therapy versus Fixed-Field IntensityModulated Radiotherapy in Radical Irradiation for Cervical Cancer without Lymphadenectasis: Dosimetric and Clinical Results[J]. Oncol Res Treat, 2018, 41(3):105-109.

6. Gall C M L , Weiden J , Eggermont L J , et al. Dendritic cells in cancer immunotherapy[J]. Nature 
Materials, 2018, 17(6):2123-2130.

7. Popovic A, Jaffee E M, Zaidi N . Emerging strategies for combination checkpoint modulators in cancer immunotherapy[J]. Journal of Clinical Investigation, 2018, 128(8):3209-3218.

8. Ribas A, Wolchok J D . Cancer immunotherapy using checkpoint blockade[J]. ence, 2018, 359(6382):13501355.

9. Dahlén, Eva, Veitonm?Ki N , Norlén, Per. Bispecific antibodies in cancer immunotherapy[J]. Therapeutic Advances in Vaccines \& Immunotherapy, 2018, 6(1):3-17.

10. June C H, O'Connor R S , Kawalekar O U, et al. CAR $\mathrm{T}$ cell immunotherapy for human cancer[J]. ence, 2018, 359(6382):1361-1365. 